\title{
GENERALIZED MEASURES WHOSE VALUES ARE OPERATORS INTO AN INTERMEDIATE SPACE ${ }^{1}$
}

\author{
BY GREGERS L. KRABBE
}

\author{
Communicated by E. J. McShane, October 2, 1961
}

The object of this note is the announcement of some results involving integration on $[-\infty, \infty]$ with respect to functions whose values are operators into an intermediate space (see Definition B). Besides several other function spaces of interest in analysis, Sobolev and Lebesgue spaces are particular types of intermediate spaces (cf., the recent contributions of CALDERón, GAGLIARDO, KREǏN, LIONS ${ }^{2}$; consequently, the present results extend previous ones dealing with Lebesgue spaces $L^{p}(a, a, \mu)$ (cf. KRABBE). Proofs and further theorems will appear elsewhere.

Each integrand will belong to the space $G_{0}$ of regulated functions ${ }^{3}$ on the interval $(-\infty, \infty)$. Let $\left[G_{0}\right]$ consist of all $g \in G_{0}$ such that $g$ coincides with its right-hand limit at each point; a Banach space $\left[G_{0}\right]$ is obtained by endowing $\left[G_{0}\right]$ with the topology of uniform convergence. Let $G_{1}$ be the family of functions of bounded variation on $(-\infty, \infty)$; in Definition $C$ we shall characterize a strictly-decreasing sequence $\left\{G_{\tau}: 0<\tau<1\right\}$ of topological spaces such that

$$
G_{0} \supset \neq G_{\imath} \supset \neq G_{\tau} \supset \neq G_{1} \quad \text { (whenever } 0<\imath<\tau<1 \text { ). }
$$

Let $E$ be a linear subset of a Banach space $\mathfrak{Y}$. If $E \mid \mathfrak{Y}$ is the normed space obtained by endowing $E$ with the norm of $\mathfrak{Y}$, then $[E, \mathfrak{Y}]$ will denote the Banach space ${ }^{4}$ of all bounded linear mappings of $E \mid \mathfrak{Y}$ into $\mathfrak{Y}$ (if $E$ is dense in $\mathfrak{Y}$, then $[E, \mathfrak{Y}]$ is identifiable with the Banach space $[\mathfrak{Y}, \mathfrak{Y}]$ of continuous linear operators in $\mathfrak{Y})$.

Our main result (Theorem $\mathrm{B}$ in $\S 1$ ) extends the following integralrepresentation property: if $g \rightarrow U g$ is a bounded linear mapping of the Banach space $\left[G_{0}\right]$ into $[E, \mathfrak{Y}]$, then the Stieltjes sums corresponding to the integral

\footnotetext{
1 This work, done while the author was on sabbatical leave at Yale University, was partially supported by a National Science Foundation grant to Purdue University.

2 An author's name in small capitals indicates a reference to the bibliography.

3 That is, $G_{0}$ consists of all the complex-valued functions $g$ having only simple discontinuities on $(-\infty, \infty)$, and such that $|g(x+0)| \neq \infty,|g(\lambda-0)| \neq \infty$ whenever $-\infty \leqq x<\infty$ and $-\infty<\lambda \leqq \infty$.

4 The topology of $[E, \mathfrak{Y}]$ is the uniform operator-topology generated by the usual operator-norm; see 1.3.
} 


$$
\int_{-\infty}^{\infty} g(\lambda) \cdot d U(\chi(-\infty, \lambda))
$$

converge to $U g$ in the (uniform operator-) topology of the Banach space $[E, \mathfrak{Y}]$; here $\chi(-\infty, \lambda)$ is the characteristic function of the interval $(-\infty, \lambda)$; note that $U(\chi(-\infty, \lambda))$ belongs to $[E, \mathfrak{Y}]$ (since it is the image of $\left.\chi(-\infty, \lambda) \in\left[G_{0}\right]\right)$. In case $g$ is a continuous function, then the Stieltjes sums converge to an ordinary Stieltjes integral.

For a moment, and before adopting the general setting of $\$ 1$, the situation will be restricted as follows. Let $E$ be the linear set of all simple functions attached to a fixed measure-space $(a, a, \mu)$; it will be convenient to set $L(p)=L^{p}(a, a, \mu)$. Suppose that $1 \leqq p_{0}<p_{1} \leqq \infty$. If $0 \leqq \iota \leqq 1$, set $\mathfrak{B}_{\iota}=L\left(p_{\imath}\right)$, where $p_{\imath}$ is defined by the equality

$$
\frac{1}{p_{\imath}}=\frac{1}{p_{0}}(1-\imath)+\frac{1}{p_{1}} \iota
$$

clearly, $p_{0} \leqq p_{\iota}<p_{\tau}<p_{1}$ whenever $0 \leqq \iota<\tau<1$.

Definition A. The expression

$$
\mathfrak{B}_{0} \leqq \mathfrak{X}<{ }_{\tau} \mathfrak{B}
$$

will mean that $\mathfrak{X}$ belongs to the family $\left\{\mathfrak{B}_{\iota}: 0 \leqq \iota<\tau\right\}$.

REMARK. The expression (2) will be retained in the more general setting of the extension 1.8 of the following proposition.

TheOREM A. Let $U$ be a bounded linear mapping of the Banach space $\left[G_{0}\right]$ into $\left[E, \mathfrak{B}_{0}\right]$, and assume that $U$ is also a bounded linear mapping of $\left[G_{0}\right]$ into the Banach space $\left[E, \mathfrak{B}_{1}\right]$. If $0<\tau<1$ and $\mathfrak{B}_{0} \leqq \mathfrak{X}<{ }_{\tau} \mathfrak{B}$, then $U$ is a continuous linear mapping of the topological space $G_{r} \cap\left[G_{0}\right]$, into $[E, \mathfrak{X}]$. Moreover, if $g \in G_{\tau} \cap\left[G_{0}\right]$, then the Stieltjes sums corresponding to the integral (1) converge to Ug in the topology of the Banach space $[E, \mathfrak{X}]$; in symbols:

$$
U g=[E, \mathfrak{X}] \lim \int g(\lambda) \cdot d U(\chi(-\infty, \lambda)) .
$$

REMARK. The mapping $U$ is necessarily an $\left[E, \mathfrak{B}_{0}\right]$-valued "measure" (terminology of BOURBAKI); but, although its values are also in $[E, \mathfrak{X}]$, the mapping $U$ need not be an $[E, \mathfrak{X}]$-valued measure (if $\left.\mathfrak{X} \neq \mathfrak{B}_{0}\right)$; for example, see Theorem $\mathrm{D}$ in [8].

Definition C. The family $W_{\alpha}$ consists of all $g \in G_{0}$ such that the sums

$$
\sum_{k=1}^{m}\left|g\left(x_{k}\right)-g\left(x_{k-1}\right)\right|^{1 / \alpha}
$$


are uniformly bounded whenever $-\infty<x_{0}<x_{1}<\cdots<x_{k-1}<x_{k}$ $<\cdots<x_{m}<\infty$. Assume $0<\tau<1$. The linear space

$$
G_{\tau}=U\left\{W_{\alpha}: \tau<\alpha \leqq 1\right\}
$$

is endowed with the inductive limit-topology of the Banach space topologies $W_{\boldsymbol{\alpha}}$.

1. Generalization to intermediate spaces. We now remove the restriction to Lebesgue spaces. The validity of Theorem A will be preserved by extending the meaning of the expression (2).

1.1. Definition. If $\mathfrak{V}$ and $\mathfrak{I}$ are topological spaces, we will say that $\mathfrak{V}$ is dominated by $\mathfrak{I}$ iff $\mathfrak{V} \subset \mathfrak{T}$ and if the identity-mapping of $\mathfrak{Y}$ into $\mathfrak{I}$ is continuous. A pair $(\mathfrak{X}, \mathfrak{Y})$ of Banach spaces is called "dominated" whenever there exists a topological Hausdorff vector space $\mathfrak{I}$ such that both $\mathfrak{X}$ and $\mathfrak{V}$ are dominated by $\mathfrak{T}$.

1.2. Hypothesis $A^{*}$. Let $\left(\mathfrak{B}_{0}, \mathfrak{B}_{1}\right)$ be a dominated pair of Banach spaces, and let $E$ be a linear subset of $\mathfrak{B}_{0} \cap \mathfrak{B}_{1}$ such that $E$ is dense in $\mathfrak{B}_{k}$ whenever $k=0,1$.

1.3. Notation. Let $\mathfrak{Z}$ be an arbitrary Banach space containing $E$; the Banach space $[E, \mathfrak{Y}]$ is endowed with the operator-norm $T \rightarrow \mathfrak{Y}[T]$.

1.4. Definition B. A space $\mathfrak{X}$ is an intermediate space if it satisfies the following three conditions

(i) the space $\mathfrak{X}$ is a Banach space containing $\mathfrak{B}_{0} \cap \mathfrak{B}_{1}$,

(ii) the pair $\left(\mathfrak{B}_{0}, \mathfrak{X}\right)$ is dominated,

(iii) there exists a number $s$ such that $0<s<1$ and

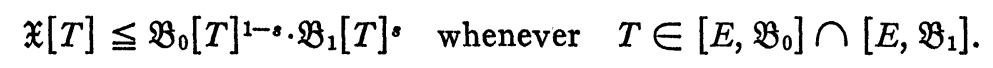

1.5. Definition $A^{*}$. Suppose $0<\tau<1$. The expression

$$
\mathfrak{B}_{0} \leqq \mathfrak{X}<\mathfrak{R}
$$

will have the following meaning: either $\mathfrak{B}_{0}=\mathfrak{X}$, or else, the following two conditions are fulfilled

(iv) there exists a semi-norm $\mathfrak{N}[\cdot]$ on the linear set $\left[E, \mathfrak{B}_{0}\right]$ $\cap\left[E, \mathfrak{B}_{1}\right]$ such that

$$
\mathfrak{R}[T] \leqq \mathfrak{B}_{0}[T]^{1-\tau} \cdot \mathfrak{B}_{1}[T]^{r} \text { whenever } T \in\left[E, \mathfrak{B}_{0}\right] \cap\left[E, \mathfrak{B}_{1}\right],
$$

(v) there exists a number $r$ with $0<r<1$ such that

$$
\mathfrak{X}[T] \leqq \mathfrak{B}_{0}[T]^{1-r} \cdot \mathfrak{N}[T]^{r} \quad \text { whenever } \quad T \in\left[E, \mathfrak{B}_{0}\right] \cap\left[E, \mathfrak{B}_{1}\right] .
$$

1.6. REMARK. The "espaces intermédiaires" defined in GagLIardo by means of functionals, and the "interpolation spaces" constructed in LIONS $[13 ; 14]$ are intermediate spaces in the sense of 1.4 . The 
following condition is called by Lions "the approximation hypothesis."

(1.6.1). There exists a sequence $\left(P_{n}\right)(n=1,2,3, \cdots)$ in $\left[\mathfrak{B}_{0}, \mathfrak{B}_{0}\right]$ $\cap\left[\mathfrak{B}_{1}, \mathfrak{B}_{1}\right]$ such that, whenever $k=0,1$ and $b_{k} \in \mathfrak{B}_{k}$, then the sequence $\left(P_{n} b_{k}\right)(n=1,2,3, \cdots)$ converges to $b_{k}$ in the topology of $\mathfrak{B}_{k}$.

1.7. EXISTENCE THEOREM. In addition to Hypothesis $\mathrm{A}^{*}$, assume that $\mathfrak{B}_{0}$ and $\mathfrak{B}_{1}$ satisfy condition (1.6.1). If $0<\tau<1$, then there exists a Banach space $\mathfrak{X}$ such that $\mathfrak{B}_{0} \leqq \mathfrak{X}<_{r} \mathfrak{B}$ and $\mathfrak{X} \neq \mathfrak{B}_{0}$.

1.8. Theorem A*. If Definition A is replaced by Definition $\mathrm{A}^{*}$, then Theorem A is true.

1.9. Remark. Condition (1.6.1) is satisfied when $\mathfrak{B}_{0}$ is the domain of the infinitesimal generator of some semi-group in $\mathfrak{B}_{1}$ (cf., Lemme 5.1 in LIONS [14]) - this is important for applications. In particular, condition (1.6.1) is satisfied when both $\mathfrak{B}_{0}$ and $\mathfrak{B}_{1}$ are Lebesgue spaces of type $L^{p}(a, Q, \mu)$; in this case, a dominating space is formed by all $(a, a, \mu)$-measurable functions, and Definition $A^{*}$ agrees with Definition $A$.

1.10. TheOREM B. Let $g \rightarrow U g$ be a bounded linear mapping of the Banach space $G_{0}$ into $\left[E, \mathfrak{B}_{0}\right]$ such that the function $\lambda \rightarrow \mathfrak{B}_{1}[U(\chi(-\infty, \lambda))]$ is bounded on $(-\infty, \infty)$. If $0<\tau<1$ and $\mathfrak{B}_{0} \leqq \mathfrak{X}<{ }_{r} \mathfrak{B}$, then the operator $g \rightarrow U \tilde{g}$ is a continuous linear mapping of the topological space $G_{\tau}$ into $[E, \mathfrak{X}]$; here $\tilde{g}$ denotes the function defined as follows:

$$
\tilde{g}(\lambda)=\lim _{\beta \rightarrow \lambda+0} g(\beta) \quad(\text { when }-\infty<\lambda<\infty) .
$$

Moreover, if $g \in G_{\tau}$, then

$$
U \tilde{g}=[E, \mathfrak{X}] \lim \int g(\lambda) \cdot d U(\chi(-\infty, \lambda)) ;
$$

compare with the conclusion (3) of Theorem A.

1.11. Remark. In several applications (for example, see 1.12), it happens that $U f=U g$ whenever $f$ and $g$ differ on a denumerable set; in this circumstance, $U \tilde{g}$ can be replaced by $U g$ throughout the statement of Theorem B.

1.12. Application. Take $L(p)=L^{p}(a, a, \mu)$, where $(a, a, \mu)$ is the measure-space of Lebesgue measure on $(-\infty, \infty)$; the linear set $E$ may be chosen to be the set of simple functions. All the hypotheses of Theorem $\mathrm{B}$ are satisfied when $U \tilde{g}$ is defined for all $x$ in $E$ by the relation $U \tilde{g}(x)=Y(g \cdot \hat{x})$, where $Y$ is the inverse operator of the Fourier transformation $x \rightarrow \hat{x}$ on $L(2)$. 
1.13. Acknowledgment. The author is indebted to Professor J. Lions for a pre-publication copy of the article LIoNs [14] - as well as for other helpful communications.

\section{REFERENCES}

N. BOURBAKI

1. Elements de mathématique, intégration, Livre VI, Chapter 6, Fascicule XXV. A. P. CALderón

2. Intermediate spaces and interpolation. (to appear)

C. FoIAs

3. Une application des distributions vectorielles à la thêorie spectrale, Bull. Sci. Math., $2^{\circ}$ série, vol. 84 (1960) pp. 147-158.

E. GAGLIARDO

4. Interpolation d'espaces de Banach et applications. (I), (II), (III), C. R. Acad. Sci. Paris, vol. 248 (1959) pp. 1912-1914, 3388-3390, 3517-3518.

5. Interpolazione di spazi di Banach e applicazioni, Richerche Mat. vol. 9 (1960) pp. 58-81.

6. Interpolazione di spazi di Banach e applicazioni, Genova, Edizioni Scientifiche, 1959, pp. 1-57.

7. Una struttura unitaria in diverse famiglie di spazi funzionali, Rend. Accad. Si. Fis. Mat. Napoli vol. 27 (1960) pp. 3-5.

G. L. KRABBE

8. Integration with respect to operator-valued functions, Bull. Amer. Math. Soc. vol. 67 (1961) pp. 214-218.

9. Integration with respect to operator-yalued functions. Acta to appear.

10. Réfractions non-hilbertiennes d'une transformation symétrique bornée, Studia Math. vol. 20 (1961) pp. 349-357.

S. G. KREǏN

11. On an interpolation theorem in operator theory, Dokl. Akad. Nauk SSSR vol. 130 (1960) pp. 491-494.

12. On the concept of a normal scale of spaces, Dokl. Akad. Nauk SSSR vol. 132 (1960) pp. 510-513.

J. L. Lions

13. Une construction d'espaces d'interpolation, C. R. Acad. Sci. Paris vol. 251 (1960) pp. 1853-1855.

14. Sur les espaces d'interpolation; dualitê. Math. Scand. vol. 9 (1961) pp. 147-177.

YALE UNIVERSITY AND

Purdue University 